\title{
Morfometría de las estructuras asociadas a la Cadena Volcánica de Los Coconucos (CVLC), Colombia
}

\author{
Wveimar Samacá ${ }^{*}$; John J. Sánchez ${ }^{2}$ \\ DOI: http://dx.doi.org/10.18273/revbol.v40n3-2018001 @ (1) \\ Forma de citar: Samacá, W., y Sánchez, J.J. (2018). Morfometría de las estructuras asociadas a la Cadena \\ Volcánica de Los Coconucos (CVLC), Colombia. Boletín de Geología, 40(3), 15-28. DOI: 10.18273/revbol. \\ v40n3-2018001.
}

\section{RESUMEN}

La Cadena Volcánica de Los Coconucos (CVLC) está formada por 14 centros volcánicos (nueve volcanes compuestos y cinco volcanes monogenéticos) a lo largo del Lineamiento de Coconucos. Dos volcanes más, los cuales son de tipo maar se ubican al SO de la CVLC. Para estudiar el desarrollo de estas estructuras, se evaluaron varios parámetros morfométricos, incluyendo elipticidad $\left(e_{i}\right)$ e irregularidad $\left(i_{i}\right)$, así como la relación alto/ancho de la base $\left(H / W_{B}\right)$ para los volcanes compuestos, y el índice $H_{c o} / W_{c o}$ para los volcanes monogenéticos. Los valores de parámetros morfométricos y las relaciones dimensionales varían así: $1,27 \leq e_{i} \leq 3,24 ; 1,12 \leq i_{i} \leq 1,53 ; 0,04 \leq H$ / $W_{B}<0,24$ y $0,02 \leq H_{c o} / W_{c o}<0,34$. Gráficos de los parámetros morfométricos ordenados de acuerdo a la posición espacial de los diferentes centros volcánicos permitieron identificar algunas tendencias parciales en la evolución en los volcanes compuestos, así como en el grado de conservación para los volcanes monogenéticos: $e_{i}$ aumenta hacia el SE; $i_{i}$ disminuye hacia el SE; $H / W_{B}$ aumenta hacia el SE y $H_{c o} / W_{c o}$ aumenta hacia el NE. En conjunto, estos valores y patrones sugieren, tanto variabilidad en aspectos endógenos y exógenos que afectan la forma de los volcanes, como posibles ciclos de actividad constructiva y afectación de la morfología y morfometría de las estructuras volcánicas.

Palabras clave: Cadena volcánica Los Coconucos - Colombia; morfometría; estructuras volcánicas; volcanes monogenéticos; volcanes compuestos.

\section{Morphometry of volcanic features associated with Los Coconucos Volcanic Chain (CVLC), Colombia}

\begin{abstract}
The Coconucos Volcanic Chain (CVLC-from its acronym in Spanish) is made up by 14 volcanic centers (nine composite volcanoes and five monogenetic volcanoes) oriented along the Coconucos Lineament. Two more centers, maar-type volcanoes, are also located to the SW, in the vicinity of the CVLC. To study the development of these volcanic centers, the ellipticity $\left(e_{i}\right)$ and irregularity $\left(i_{i}\right)$ morphometric parameters as well as the base-toheight $H / W_{B}$ for composite volcanoes and the height-of-cone to width-of-cone $H_{c o} / W_{c o}$ ratios for monogenetic centers, were evaluated. The values for morphometric parameters and dimensional ratios vary as follows: $1.27 \leq e_{i} \leq 3.24 ; 1.12 \leq i_{i} \leq 1.53 ; 0.04 \leq H / W_{B} \leq 0.24$ and $0.02 \leq H_{c o} / W_{c o} \leq 0.34$. Graphs of morphometric parameters ordered according to the spatial position of the volcanic centers allowed identification of partial tendencies in the evolution of composite volcanoes and patterns in the state of preservation for monogenetic centers: $e_{i}$ increases to the SE; $i_{i}$ decreases to the SE; $H / W_{B}$ increases to the SE and $H_{c o} / W_{c o}$ increases to the NE. Together, these values and patterns suggest not only variability of endogenous and exogenous processes that affect the shapes of volcanoes, but also possible cycles of constructive activity and affectation of morphology and morphometry of the volcanic structures.
\end{abstract}

Keywords: Coconucos volcanic chain - Colombia; morphometry; volcanic structures; monogenetic volcanoes; composite volcanoes.

\footnotetext{
${ }^{1}$ Departamento de Geociencias, Facultad de Ciencias, Universidad Nacional de Colombia, Bogotá, Colombia.

(*)wsamacat@unal.edu.co

${ }^{2}$ Departamento de Geociencias y Medio Ambiente, Facultad de Minas, Universidad Nacional de Colombia, Medellín,

Colombia.jjsanchezag@unal.edu.co
} 


\section{INTRODUCCIÓN}

La determinación de parámetros morfométricos aplicados a estructuras volcánicas se ha efectuado desde mediados del siglo XX, para estudiar los procesos evolutivos y los tipos específicos de estructuras volcánicas. Cabe destacar de la extensa literatura existente sobre este tema los trabajos realizados por Porter (1972) y Wood (1980) en volcanes monogenéticos individuales, y aquellos asociados a un volcán poligenético (Favalli et al., 2009) o relacionados a campos de volcanes monogenéticos extensos (Settle, 1979). Para el caso de volcanes poligenéticos, se destacan los análisis realizados a volcanes individuales
(Karátson et al., 2010) o a nivel regional (Grosse et al., 2009; 2012), con el fin de definir clasificaciones de acuerdo a la forma de las estructuras.

En la Cadena Volcánica de Los Coconucos (CVLC), ubicada aproximadamente a $20 \mathrm{~km}$ al SE de la ciudad de Popayán (Departamento del Cauca, Colombia) existen 14 volcanes monogenéticos y poligenéticos alineados en orientación preferencial NW-SE (FIGURA 1), siguiendo el trazo principal del Lineamiento de Coconucos (Monsalve, 2000). Los rasgos más relevantes publicados acerca de los centros volcánicos de la CVLC se resumen en la TABLA 1.

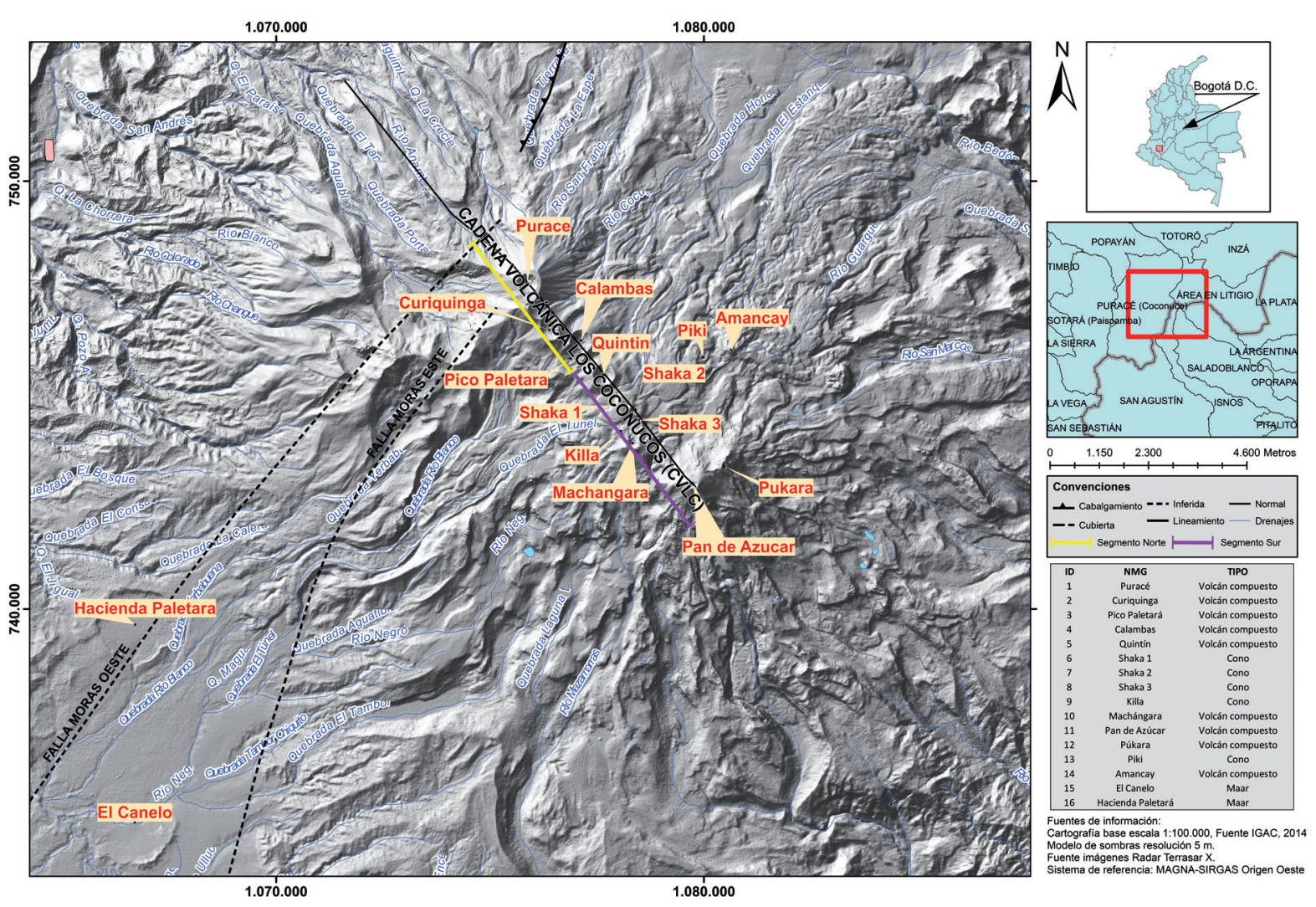

FIGURA 1. Ubicación de la Cadena Volcánica de Los Coconucos (CVLC) y estructuras volcánicas asociadas.

La CVLC es considerada evidencia de resurgencia magmática de la denominada Caldera de Paletará (Torres et al., 1995; Robertson et al., 2002; Samacá, 2016) y en las últimas décadas ha recibido especial atención debido a la actividad reciente de su centro volcánico más septentrional, el volcán Puracé (Monsalve et al., 2014). Sin embargo, no muchos trabajos se han enfocado en la parametrización morfométrica y la evolución de las geoformas asociadas, por lo que el objetivo central de la presente investigación es contribuir con datos precisos acerca de índices morfométricos y morfología de todos los centros volcánicos de la CVLC y algunas otras estructuras cercanas. 
TABLA 1. Estructuras volcánicas asociadas a la CVLC (recopilado de Flórez, 1983; Robertson et al., 2002; Flórez, 2003; INGEOMINAS, 2003).

\begin{tabular}{|c|c|c|c|c|c|c|}
\hline Volcán & $\begin{array}{l}\text { Latitud } \\
\left({ }^{\circ}\right)\end{array}$ & $\begin{array}{l}\text { Longitud } \\
\left({ }^{\circ}\right)\end{array}$ & Tipo & $\begin{array}{c}\text { Nivel de } \\
\text { Actividad }\end{array}$ & Tipo de depósitos & Observaciones \\
\hline Puracé & 2,3137 & 76,3954 & $\begin{array}{l}\text { Volcán } \\
\text { compuesto }\end{array}$ & $\begin{array}{l}\text { Activo } \\
\text { (erupciones } \\
\text { recientes) }\end{array}$ & $\begin{array}{c}\text { Flujos de lava, Corrientes de } \\
\text { Densidad Piroclástica (CDP), } \\
\text { lahares. }\end{array}$ & $\begin{array}{l}\text { Sin indicios de } \\
\text { erosión glaciar }\end{array}$ \\
\hline Curiquinga & 2,3037 & 76,3908 & $\begin{array}{l}\text { Volcán } \\
\text { compuesto }\end{array}$ & Latente & $\begin{array}{l}\text { Flujos de lava en bloques y } \\
\text { flujos piroclásticos con pómez, } \\
\text { escoria, bombas y líticos. }\end{array}$ & - \\
\hline Pico Paletará & 2,2977 & 76,3864 & $\begin{array}{l}\text { Volcán } \\
\text { compuesto }\end{array}$ & Latente & $\begin{array}{c}\text { Flujos de lava provenientes } \\
\text { de los cráteres Curiquinga y } \\
\text { Quintín. }\end{array}$ & - \\
\hline Calambas* & 2,3015 & 76,3841 & $\begin{array}{l}\text { Volcán } \\
\text { compuesto }\end{array}$ & - & $\begin{array}{c}\text { Flujos de lava masivos } \\
\text { hidrotermalizados; cono } \\
\text { cubierto con ceniza y bombas } \\
\text { recientes }\end{array}$ & $\begin{array}{c}\text { Comparte parte } \\
\text { de su estructura } \\
\text { principal con Pico } \\
\text { Paletará }\end{array}$ \\
\hline Quintín & 2,2942 & 76,3794 & $\begin{array}{l}\text { Volcán } \\
\text { compuesto }\end{array}$ & Latente & $\begin{array}{l}\text { Depósitos volcaniclásticos, } \\
\text { CDP. }\end{array}$ & $\begin{array}{l}\text { Los productos } \\
\text { están cubiertos por } \\
\text { lavas y productos } \\
\text { de otros volcanes. }\end{array}$ \\
\hline Shaka 1 & 2,2877 & 76,3748 & Cono & Latente & $\begin{array}{c}\text { Flujos de lava en bloque, } \\
\text { cubiertas por depósitos de } \\
\text { ceniza }\end{array}$ & $\begin{array}{l}\text { Predominio de } \\
\text { coladas lávicas } \\
\text { del Holoceno; } \\
2 \text { estructuras } \\
\text { asociadas }\end{array}$ \\
\hline Shaka 2 & 2,2869 & 76,3727 & Cono & - & $\begin{array}{l}\text { Flujos de lava, cubiertas por } \\
\text { depósitos de ceniza }\end{array}$ & $\begin{array}{l}\text { Cono asociado a } \\
\text { Shaka } 1\end{array}$ \\
\hline Shaka 3 & 2,2845 & 76,3718 & Cono & - & $\begin{array}{l}\text { Flujos de lava, cubiertas por } \\
\text { depósitos de ceniza }\end{array}$ & $\begin{array}{l}\text { Cono asociado a } \\
\text { Shaka } 1\end{array}$ \\
\hline Killa* & 2,2823 & 76,3759 & Cono & - & & $\begin{array}{l}\text { Remanente de } \\
\text { aguja; estructura } \\
\text { columnar hacia el } \\
\text { centro y laminar } \\
\text { hacia los bordes }\end{array}$ \\
\hline Machangara & 2,2798 & 76,3733 & $\begin{array}{l}\text { Volcán } \\
\text { compuesto }\end{array}$ & Extinto & $\begin{array}{l}\text { Flujos de lava masivos con } \\
\text { estructura cordada }\end{array}$ & - \\
\hline $\begin{array}{l}\text { Pan de } \\
\text { Azúcar }\end{array}$ & 2,2710 & 76,3605 & $\begin{array}{l}\text { Volcán } \\
\text { compuesto }\end{array}$ & Latente & $\begin{array}{l}\text { Flujos de lava en bloque, } \\
\text { textura porfirítica }\end{array}$ & $\begin{array}{l}\text { Presenta un cono } \\
\text { adventicio }\end{array}$ \\
\hline Pukara & 2,2749 & 76,3541 & $\begin{array}{l}\text { Volcán } \\
\text { compuesto }\end{array}$ & Extinto & $\begin{array}{l}\text { Flujos de lava fracturados } \\
\text { y alterados; fragmentos de } \\
\text { escoria }\end{array}$ & - \\
\hline Piki* & 2,2980 & 76,3584 & Cono & - & $\begin{array}{l}\text { Flujos de lava de textura } \\
\text { porfirítica, cubiertas en sectores } \\
\text { por productos de otros volcanes }\end{array}$ & $\begin{array}{l}\text { Edificio en forma } \\
\text { abombada }\end{array}$ \\
\hline Amancay & 2,2994 & 76,3525 & $\begin{array}{c}\text { Volcán } \\
\text { compuesto }\end{array}$ & - & $\begin{array}{l}\text { Flujos de lava en bloque, } \\
\text { macro cordadas }\end{array}$ & $\begin{array}{l}\text { Morfología del } \\
\text { edificio bien } \\
\text { conservada }\end{array}$ \\
\hline
\end{tabular}

*Estructuras volcánicas de difícil identificación, de acuerdo a los autores mencionados en la recopilación. 


\section{MARCO TEÓRICO}

Considerando factores como la historia eruptiva y la complejidad de la estructura, las estructuras volcánicas asociadas a la CVLC pueden definirse siguiendo la clasificación de De Silva y Lindsay (2015), así:

- Volcanes poligenéticos: Son estructuras que han tenido varios ciclos eruptivos a través de su historia, por lo que son considerados grandes estructuras construidas a lo largo de decenas o cientos de miles de años; dentro de este grupo se identifican los volcanes compuestos (equivalente al término "estratovolcanes", comúnmente utilizado en geomorfología y vulcanología), los volcanes escudo y las calderas.

- Volcanes monogenéticos: Estas estructuras se han formado durante un ciclo eruptivo único; los episodios pueden ser de corta duración (semanas a meses) y pueden continuar por períodos sucesivos manifestados durante algunos años o décadas. De acuerdo a estos autores, se incluyen dentro de esta categoría los conos piroclásticos y de escoria, los maares, los anillos piroclásticos (tuff rings) y los domos de composición silícea.

Índices morfométricos de volcanes compuestos: Grosse et al. (2009) plantean algunos índices morfométricos generados a partir de las curvas de nivel. Dos de estos índices definen la similitud a dos tipos de contornos independientes: la elongación y la irregularidad, que sumados indican la forma en planta (plan shape) de un volcán compuesto:
- Índice de elipticidad $\left(e_{i}\right)$, que cuantifica la elongación de los contornos:

$$
e_{i}=\frac{\pi \times(L / 2)^{2}}{A}
$$

Donde $L$ es la longitud del eje principal del contorno, y $A$ es el área que encierra el contorno. Si $e_{i} \sim 1$, el contorno tiene forma de círculo; si $e_{i} \gg 1$, el contorno es elongado. El promedio de los valores $e_{i}$ de los contornos es la elongación de la estructura en planta.

- Índice de irregularidad $\left(i_{i}\right)$, que cuantifica la irregularidad o complejidad relacionando el perímetro y el área del contorno. De acuerdo a Grosse et al. (2009), primero debe calcularse para el contorno el índice de disección $d_{i}$ :

$$
d_{i}=\frac{P}{2 \times A} \times \sqrt{A / \pi}
$$

Donde $P$ es el perímetro del contorno y $A$ es el área que encierra el contorno. Debido a que el índice de disección en este sentido es aproximadamente dependiente de la elipticidad del contorno, los autores introducen el índice de irregularidad $\left(i_{i}\right)$ :

$$
i_{i}=d_{i(\text { contorno })}-d_{i(\text { elipse })}
$$

Otros parámetros sugeridos por los autores, que sirven para identificar la forma en perfil (profile shape) de los volcanes compuestos son la pendiente (total, pendiente de los flancos y pendiente media máxima), junto con las relaciones altura del volcán/ancho de la base $(H /$ $\left.W_{B}\right)$ y ancho de la cima/ancho de la base $\left(W_{S} / W_{B}\right)$ (FIGURA 2A).
A.

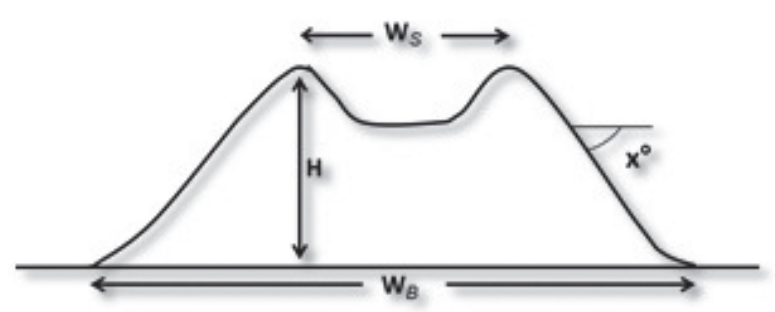

B.

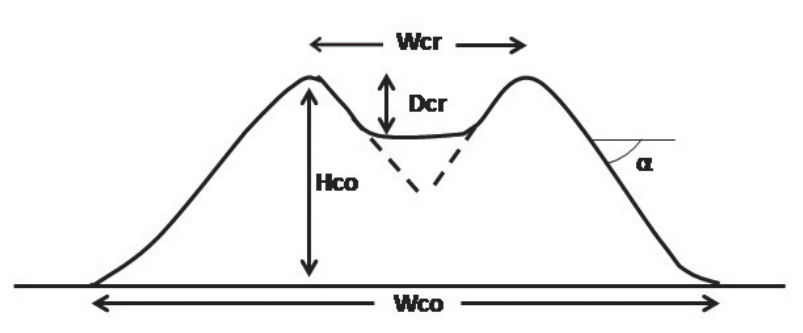

FIGURA 2. A. Parámetros morfométricos de la forma en perfil de volcanes compuestos (Grosse et al., 2009). H=Altura del volcán; $W_{s}=$ Ancho de la cima (width of summit); $W_{B}=$ Ancho del volcán (width of base); $x^{o}=$ Pendiente. B. Parámetros morfométricos (Porter, 1972) para volcanes monogenéticos. $H_{c o}=$ altura del cono; $W_{c r}=$ ancho del cráter; $D_{c r}=$ profundidad del cráter (depth of crater); $W_{c o}=$ ancho del cono; $\alpha=$ pendiente. 
La clasificación morfométrica de volcanes compuestos de Grosse et al. (2009) define tres categorías:

- Cónicos: Los volcanes compuestos cónicos tienen una forma simple, teniendo un perfil suavemente cóncavo, con una forma en planta circular o semicircular (bajos valores de $e_{i}$ ) y regular (bajos valores de $i_{i}$ ). Algunas estructuras de este tipo estudiadas por los autores exhiben $H / W_{B}>0,15$, las pendientes de los flancos oscilan entre $21 \mathrm{y}$ $34^{\circ}$, y la pendiente máxima entre 27 y $37^{\circ}$.

- Sub-cónicos: Para los volcanes compuestos en esta categoría, $0,10 \leq H / W_{B} \leq 0,16$. En cuanto a los demás índices, los valores son muy variables, pero se encuentran en una posición intermedia con respecto a las demás categorías. Para los autores, es posible que los volcanes compuestos sub-cónicos sean estructuras morfométricamente transicionales.

- Macizos: Estas estructuras presentan valores $H /$ $W_{B}<0,10$ y pendiente media de los flancos inferior a $20^{\circ}$; son irregulares y usualmente tienden a ser elípticos. A diferencia de los volcanes compuestos cónicos y sub-cónicos, la mayoría de los volcanes compuestos de tipo macizo incluyen un espectro de volumen que va hasta $90 \mathrm{~km}^{3}$.

Adicionalmente Grosse et al. (2009) sugieren que, de acuerdo a los valores de los índices, los volcanes compuestos con formas simples pueden seguir la tendencia evolutiva cono $\rightarrow$ sub-cono $\rightarrow$ macizo.

Índices morfométricos de volcanes monogenéticos: De acuerdo a Porter (1972) los parámetros morfométricos aplicables a este tipo de volcanes son: $H_{c o}$ (altura del cono); $W_{c o}$ (ancho basal del cono); $W_{c r}^{c o}$ (ancho del cráter); $D_{c r}^{c o}$ (profundidad del cráter) y $\alpha$ (ángulo máximo de pendiente, usualmente en el rango $25^{\circ} \pm 2^{\circ}$ ). Porter (1972) y Settle (1979) proponen que uno de los principales índices es la relación $H_{c o} / W_{c o}$ (FIGURA 2B), que reflejaría, en primera aproximación, el ángulo de reposo de los flancos de los conos (volcanes monogenéticos) constituidos por depósitos piroclásticos o tefras. Los factores que influyen en el comportamiento del índice $H_{c o} / W_{c o}$ son principalmente: la naturaleza de piroclastos y su forma de depositación, la naturaleza de los flujos de lava, la acción de agentes exógenos (ejemplo: meteorización y erosión) y la inestabilidad de la pendiente.

\section{MATERIALES Y MÉTODOS}

Utilizando un Modelo Digital de Elevación (DEM, por sus siglas en inglés) con resolución espacial de 5 $\mathrm{m} /$ píxel generado a partir de imágenes de radar sensor Terrasar X (imágenes cortesía del Banco de Imágenes del Instituto Geográfico Agustín Codazzi-IGAC), se calcularon los índices morfométricos para cada una de las estructuras volcánicas en la CVLC y su vecindad, siguiendo los procedimientos resumidos a continuación:

- Morfometría de volcanes compuestos: Mediante el código IDL MORVOLC (Grosse et al., 2012) se obtienen, a partir del DEM, los parámetros morfométricos para volcanes compuestos $\left(e_{i}\right.$ e $i_{i}$ promediados para cada volcán compuesto). Una vez calculados, estos índices se pueden analizar individualmente para cada volcán, o en conjunto, para evaluar tendencias y realizar comparaciones y así plantear posibles factores que influyen en las características morfológicas (por ejemplo, la proximidad con otros volcanes compuestos o monogenéticos y los productos particulares de cada centro). Igualmente, con el DEM se midieron los parámetros que definen la forma en perfil de los volcanes.

- Morfometría de volcanes monogenéticos: El cálculo de índices morfométricos para los volcanes monogenéticos, se realizó así: 1) Recorte del DEM de acuerdo a la superficie de cada estructura y 2) Cálculo de la relación $H_{c o} / W_{c o}$ para cada estructura a partir de los valores del DEM. Se utilizó el software ArcGIS y por completitud, se realizaron también los cálculos de la relación $H_{c o} / W_{c o}$ en forma manual.

Para facilitar el análisis de los datos e identificar tendencias se grafican los valores de los diferentes parámetros ordenados de acuerdo a la posición espacial del centro volcánico: Si se indica que el ordenamiento es en sentido N-S esto implica que los valores del parámetro bajo análisis se grafican de acuerdo a la coordenada de latitud del volcán correspondiente, empezando por los más septentrionales (parte izquierda del eje horizontal del gráfico) y terminando por los más meridionales (parte derecha del eje horizontal del gráfico). Por otra parte, si se indica que el ordenamiento es en sentido E-W esto implica que los valores del parámetro bajo análisis se grafican de acuerdo a la coordenada de longitud del volcán, 
empezando por los volcanes que están ubicados más al oriente (parte izquierda del eje horizontal del gráfico) y terminando por los que están ubicados más al occidente (parte derecha del eje horizontal del gráfico). Esta metodología ha sido aplicada exitosamente en otras investigaciones, principalmente acerca de sismos volcánicos y es sugerida aquí como una herramienta sencilla de análisis de índices.

\section{RESULTADOS}

Morfometría de volcanes compuestos: Los parámetros morfométricos para los volcanes compuestos se muestran en la TABLA 2. Los valores de $e_{i}$ varían en el rango 1,269-3,236. Los volcanes Púkara, Quintín, Machángara y Calambas exhiben, en conjunto, mayor elongación e irregularidad que los volcanes Curiquinga, Pan de Azúcar, Amancay, Pico Paletará y Puracé (FIGURA 1). Este resultado está basado en la media y la mediana de $e_{i}$ e $i_{i}$ como medidas de tendencia central en cada uno de estos conjuntos de volcanes. Al graficar los valores de $e_{i}$ siguiendo un sentido N-S (con respecto a la coordenada de latitud de cada volcán compuesto, primero los volcanes más septentrionales y por último los más meridionales) se observa carácter oscilante con tendencia creciente incipiente de $e_{i}$ de norte a sur (FIGURA 3A). De modo similar, si se ordenan los valores de $e_{i}$ siguiendo un sentido E-W (con respecto a la coordenada de longitud de cada volcán, primero los volcanes más orientales y por último los más occidentales), se observa oscilación con tendencia creciente incipiente en sentido W-E, (FIGURA 3C). Así, de modo general, la elipticidad aumenta hacia el sector SE de la CVLC. Los valores de $i_{i}$ varían en el rango 1,115-1,530 y exhiben más estabilidad y tendencia decreciente incipiente hacia el sur (FIGURA 3B). Al graficar los valores de $i_{i}$ siguiendo un sentido E-W, se observa tendencia parcial decreciente hacia el E (FIGURA 3D). La irregularidad de los volcanes de la CVLC decrece de manera aproximada hacia el SE.
A.

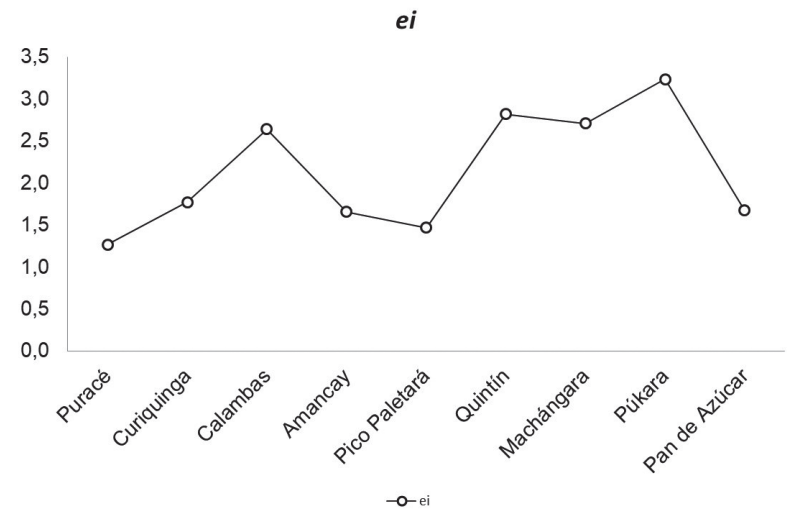

C.

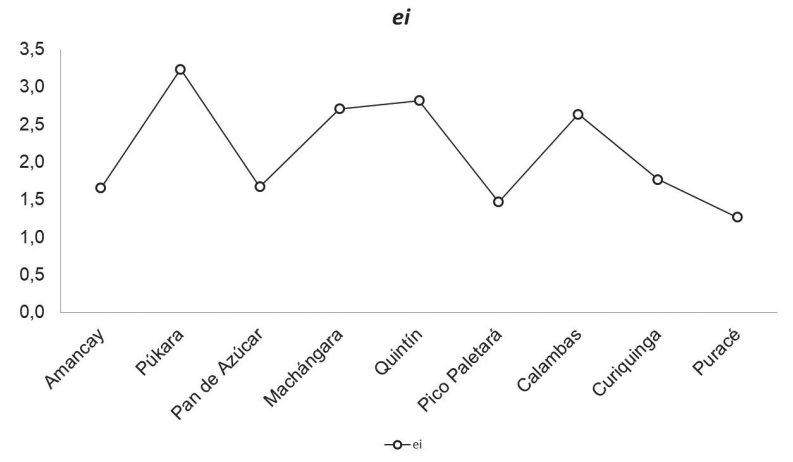

B.

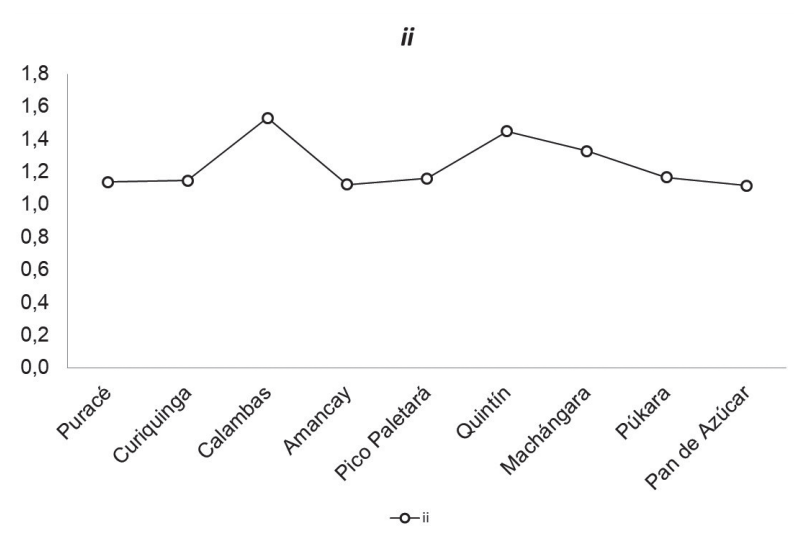

D.

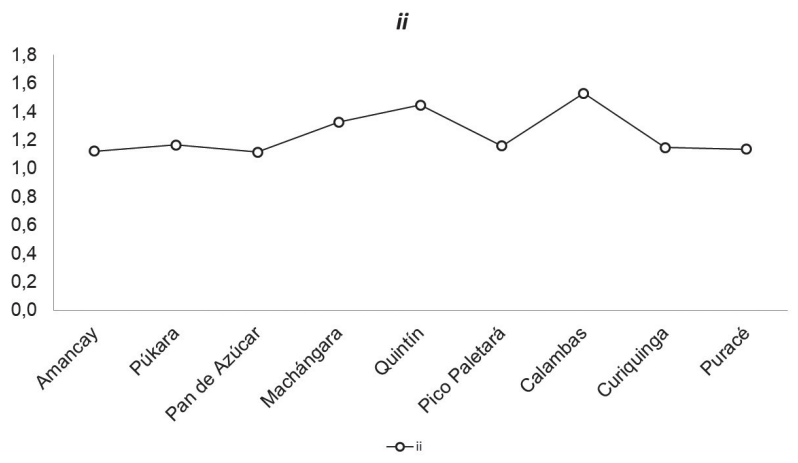

FIGURA 3. Gráficos de los valores de $e_{i}$ e $i_{i}$ para volcanes compuestos de la CVLC. A y B. Valores ordenados de acuerdo a la coordenada de latitud (los más septentrionales a la izquierda y los más meridionales a la derecha). C y $\mathbf{D}$. Valores ordenados de acuerdo a la coordenada de longitud (los más orientales a la izquierda y los más occidentales a la derecha). 


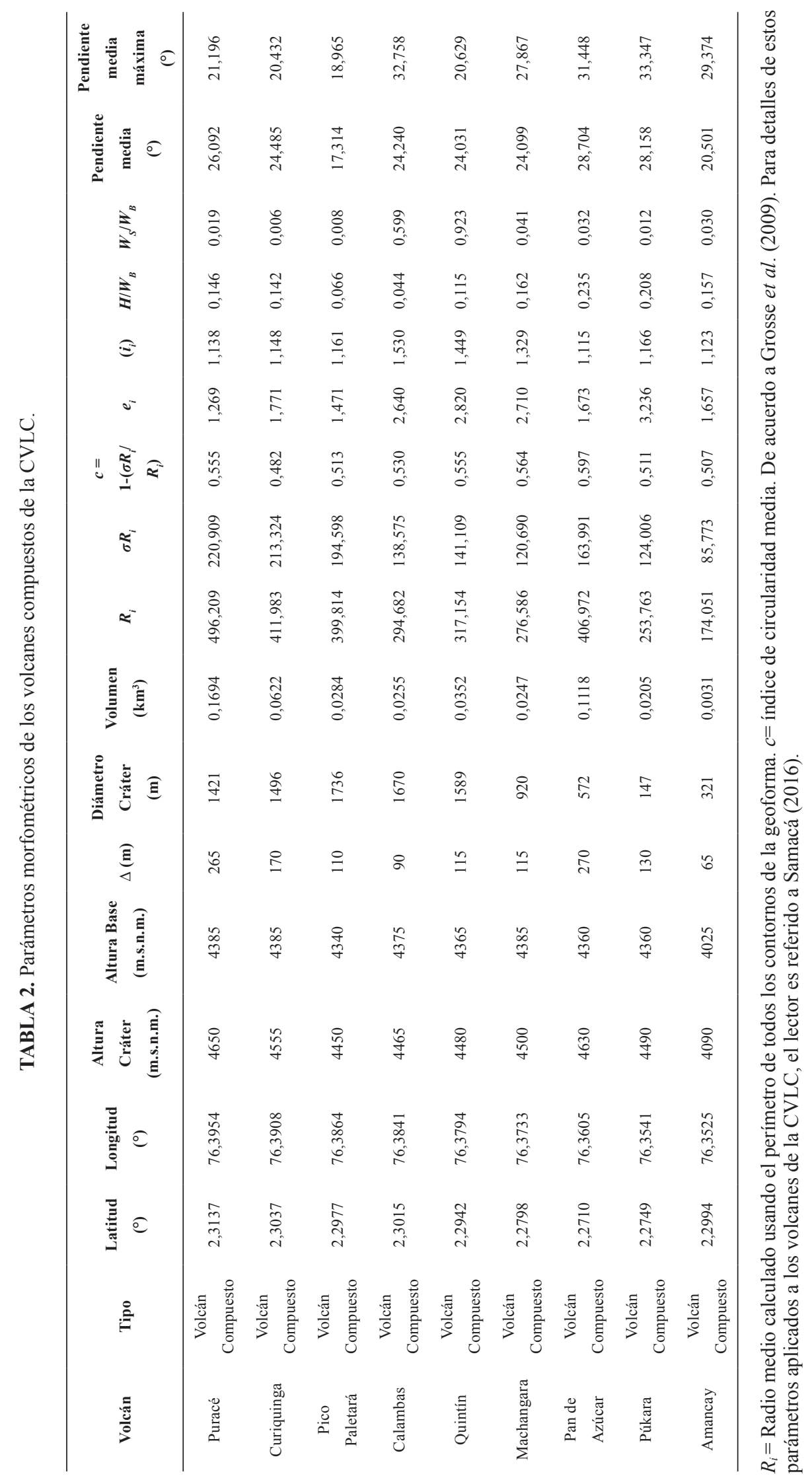


En lo referente a la forma en perfil, al ordenar los valores de $H / W_{B}$ en sentido N-S, hay oscilación y tendencia creciente de este valor hacia el sur (FIGURA 4A). La gráfica de $H / W_{B}$ con los valores ordenados de acuerdo a la longitud, revela que hay un patrón oscilante, con marcada tendencia a aumentar hacia el E (FIGURA 4B). De modo general, la relación $H / W_{B}$ crece hacia el SE de la CVLC.
A.

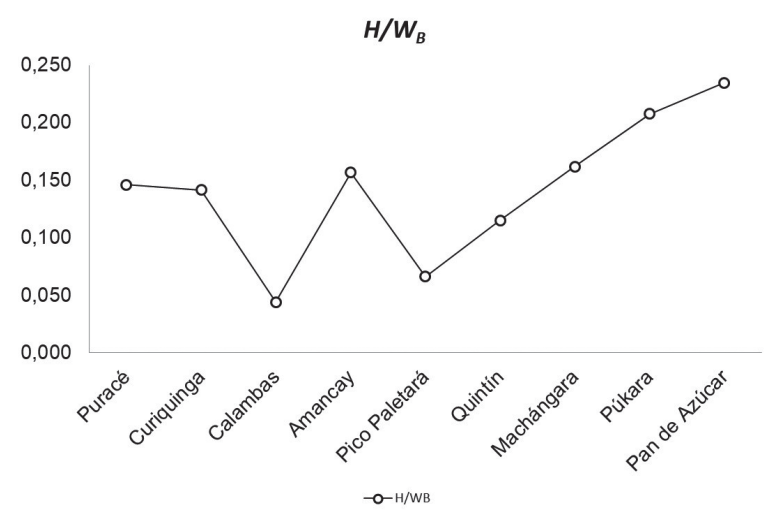

B.

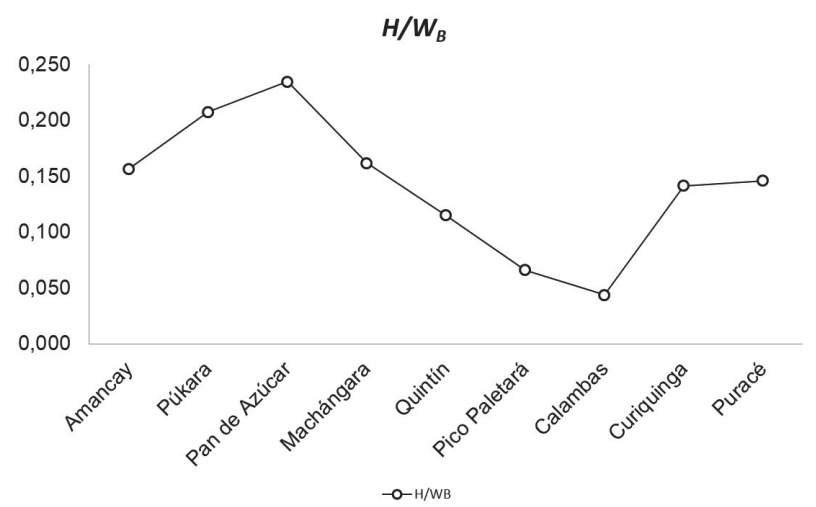

FIGURA 4. Gráficos de los valores de $H / W_{B}$ para volcanes compuestos de la CVLC. A. Valores ordenados de acuerdo a la coordenada de latitud. B. Valores ordenados de acuerdo a la coordenada de longitud. Convenciones de orden como en la FIGURA 3.

Morfometría de volcanes monogenéticos: Los valores de parámetros morfométricos para este grupo, que incluye los conos Shaka 1, Shaka 2, Shaka 3, Killa y Piki y los maares Hacienda Paletará y El Canelo, se muestran en la TABLA 3. La relación $H_{c o} / W_{c o}$ varía en el rango 0,017-0,340. El gráfico de la relación $H_{c o} / W_{c o}$ de acuerdo a la latitud del volcán muestra que, con excepción de los conos Shaka 2 y Shaka 3, hay un decremento aproximadamente gradual de la relación hacia el sur. Al ordenar los valores de $H_{c o} / W_{c o}$ de acuerdo a la coordenada de longitud, se revela una progresión a valores mínimos hacia el W. De modo general, para los volcanes monogenéticos de la CVLC y sus vecindades, el valor de $H_{c o} / W_{c o}$ disminuye hacia el SW (FIGURA 5).

TABLA 3. Parámetros morfométricos calculados para volcanes monogenéticos de la CVLC y cercanos a ésta.

\begin{tabular}{|c|c|c|c|c|c|c|c|c|c|c|c|c|c|c|}
\hline Volcán & Tipo & $\begin{array}{l}\text { Latitud } \\
\left({ }^{\circ}\right)\end{array}$ & $\begin{array}{l}\text { Longitud } \\
\left({ }^{\circ}\right)\end{array}$ & $\begin{array}{c}\text { Altura } \\
\text { Basal } \\
\text { (m.s.n.m.) }\end{array}$ & $\begin{array}{c}\text { Altura } \\
\text { Cráter } \\
\text { (m.s.n.m.) }\end{array}$ & $\begin{array}{c}\text { Altura } \\
\text { Cono, } \\
H_{c o}\left(A_{c o}\right)\end{array}$ & $\begin{array}{c}\text { Diámetro } \\
\text { Basal, } \\
W_{c o}\left(D_{c o}\right)\end{array}$ & $\begin{array}{c}\text { Diámetro } \\
\text { de } \\
\text { Cráter, } \\
W_{c r}\left(D_{c r}\right) \\
\end{array}$ & $\begin{array}{c}\text { Profundidad } \\
\text { de Cráter } \\
(\mathrm{m}), D_{c r}\left(P_{c r}\right)\end{array}$ & $\begin{array}{c}\text { Radio } \\
\text { Basal } \\
\text { (m) }\end{array}$ & $\begin{array}{c}\text { Radio } \\
\text { Cráter } \\
\text { (m) }\end{array}$ & $\begin{array}{c}\text { Volumen } \\
\text { Estimado } \\
\left(\mathbf{k m}^{3}\right)\end{array}$ & $\mathrm{H}_{\mathrm{co}} / \mathrm{W}_{\mathrm{co}}$ & $\begin{array}{c}\text { Pendiente } \\
\text { Máxima } \\
\left({ }^{\circ}\right)\end{array}$ \\
\hline Shaka 1 & Cono & 2,2877 & 76,3748 & 4380 & 4450 & 70 & 761,8 & 226,6 & 20 & 380,90 & 113,30 & 0,0147 & 0,092 & 39,7 \\
\hline Shaka 2 & Cono & 2,2869 & 76,3727 & 4350 & 4410 & 60 & 439,1 & 235,7 & 5 & 219,57 & 117,83 & 0,0055 & 0,137 & 39,4 \\
\hline Shaka 3 & Cono & 2,2845 & 76,3718 & 4355 & 4395 & 40 & 210,8 & 100,3 & 10 & 105,41 & 50,14 & 0,0008 & 0,190 & 46,4 \\
\hline Killa & Cono & 2,2823 & 76,3759 & 4370 & 4390 & 20 & 377,7 & 23,0 & 20 & 188,86 & 11,52 & 0,0008 & 0,053 & 63,8 \\
\hline Piki & Cono & 2,2980 & 76,3584 & 4010 & 4100 & 90 & 264,9 & 99,6 & 15 & 132,44 & 49,80 & 0,0025 & 0,340 & 44,3 \\
\hline $\begin{array}{l}\text { Hacienda } \\
\text { Paletará }\end{array}$ & Maar & 2,2415 & 76,4779 & 3000 & 3040 & 40 & 2322,3 & 1812,9 & 20 & 1161,14 & 906,43 & 0,1350 & 0,017 & 24 \\
\hline $\begin{array}{l}\text { El Canelo } \\
\text { (1) }\end{array}$ & Maar & 2,1998 & 76,4779 & 3050 & 3135 & 85 & 2378,4 & 1941,1 & 50 & 1189,22 & 970,56 & 0,3125 & 0,036 & 33 \\
\hline
\end{tabular}

(1) Valores aproximados por el estado de degradación. De acuerdo a Porter (1972). Para detalles de estos parámetros aplicados a los volcanes monogenéticos de la CVLC, el lector es referido a Samacá (2016). 
A. Hco/Wco

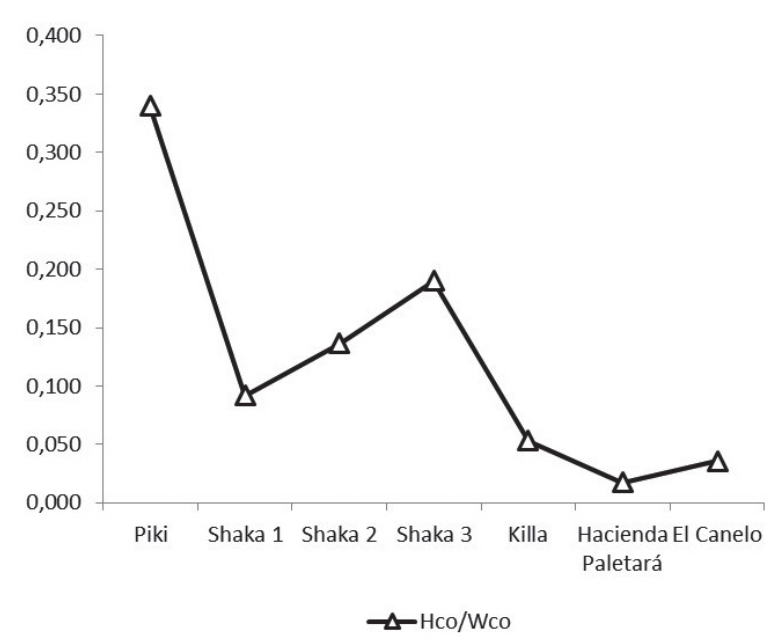

B.

Hco/Wco

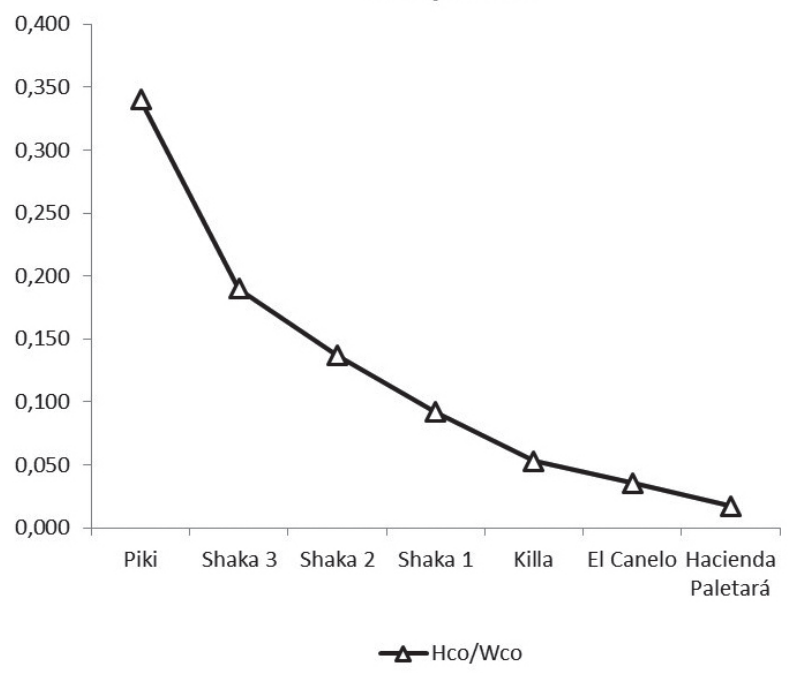

FIGURA 5. Gráficos de los valores de $H_{c o} / W_{c o}$ para volcanes monogenéticos de la CVLC. A. Valores ordenados de acuerdo a la coordenada de latitud. B. Valores ordenados de acuerdo a la coordenada de longitud. Convenciones de orden como en la FIGURA 3.

\section{DISCUSIÓN}

Los índices de elipticidad $\left(e_{i}\right)$ e irregularidad $\left(i_{i}\right) \mathrm{y}$ la relación $H / W_{B}$, determinados para los volcanes compuestos de la CVLC (FIGURA 1, TABLA2) han sido analizados ordenando los valores calculados de acuerdo a la latitud y longitud (FIGURA 3). Esto permitió revelar que la forma en planta de los volcanes se hace más elíptica y menos irregular hacia el sector SE de la CVLC, lo cual sugiere en primera aproximación diferencias en la complejidad de las estructuras y posiblemente diferencias en los sistemas de conductos subvolcánicos. Del mismo modo, la forma en perfil (cuantificada por la relación $H / W_{B}$, FIGURA 4) varía más o menos de modo oscilante, pero en general los volcanes son más cónicos hacia el sector SE de la CVLC.

En particular, los volcanes compuestos del sur de la CVLC (Machángara, Pan de Azúcar) tienden a presentar forma cónica con morfologías conservadas y las estructuras más desarrolladas. Considerando sus alturas y volúmenes, los volcanes Puracé y Pan de Azúcar, ubicados en los extremos NW y SE de la CVLC, respectivamente, exhiben formas bien preservadas. También hay volcanes compuestos menos desarrollados (Amancay) o que han sido afectados morfológicamente por procesos erosivos (Calambas y el volcán compuesto tipo macizo Púkara). Lo anterior denota que, a pesar que los parámetros morfométricos pueden aproximar el desarrollo o la tendencia evolutiva de un grupo de volcanes compuestos, hay comportamientos y procesos geomorfológicos particulares para cada volcán. Los índices morfométricos son entonces complementarios a la caracterización geomorfológica que está a su vez relacionada a la morfogénesis y a la morfodinámica.

Las diferencias en morfología estarían relacionadas a variaciones en aspectos endógenos y exógenos que modulan la evolución de los centros volcánicos (para volcanes compuestos, se sugiere a Karátson et al., 2010; en Porter, 1972 y Wood, 1980 se tratan algunos casos de volcanes monogenéticos). Dentro de los aspectos endógenos se puede concebir que existan variaciones temporales en la actividad magmática o complejidades espaciales en el sistema superficial de conductos, que pueden llevar a diferentes regímenes y formas de salida y emplazamiento de los productos volcánicos, que se hacen más complejas con el tiempo a medida que el magma busca nuevos caminos para llegar a la superficie. Esto es interesante porque, aunque el Lineamiento de los Coconucos es la estructura mayor a lo largo de la cual muchos de los volcanes en la CVLC han llevado sus productos a superficie, las particularidades morfológicas quizá se relacionan a detalles de cada centro eruptivo.

Dentro de los aspectos exógenos es razonable suponer que variaciones espaciales y temporales en patrones de estado del tiempo y clima influencian la meteorización y erosión diferencial de los volcanes 
ubicados en diferentes áreas (Wood, 1980). El tipo de vulcanismo y la naturaleza de los productos también son factores que influencian la forma y la preservación de un edificio volcánico. Los volcanes compuestos del sur de la CVLC tienen morfologías conservadas y de constitución reciente y exhiben características similares en sus productos (TABLA 1).

Estudios previos han caracterizado los centros volcánicos de la CVLC y vecindades en las categorías de volcanes compuestos y conos (TABLA 1), sin embargo, la caracterización de la morfología mediante la cuantificación morfométrica aquí presentada posibilita una clasificación más elaborada. Aplicando la metodología de Grosse et al. (2012), se encuentra que la CVLC incluye volcanes compuestos y monogenéticos agrupables en subconos, conos y un macizo (FIGURA 6). Púkara es el único volcán compuesto cuya morfología se acerca a la categoría de macizo y presenta rasgos que indican algo de degradación en su estructura; Calambas y Amancay tienen poca altitud y bajo volumen, por lo que pueden catalogarse como estructuras poco evolucionadas, aunque las características morfológicas de Calambas indican también un volcán que presenta rasgos de degradación (cráter con apertura muy amplia y estructura parcialmente influenciada por la actividad de Pico Paletará).

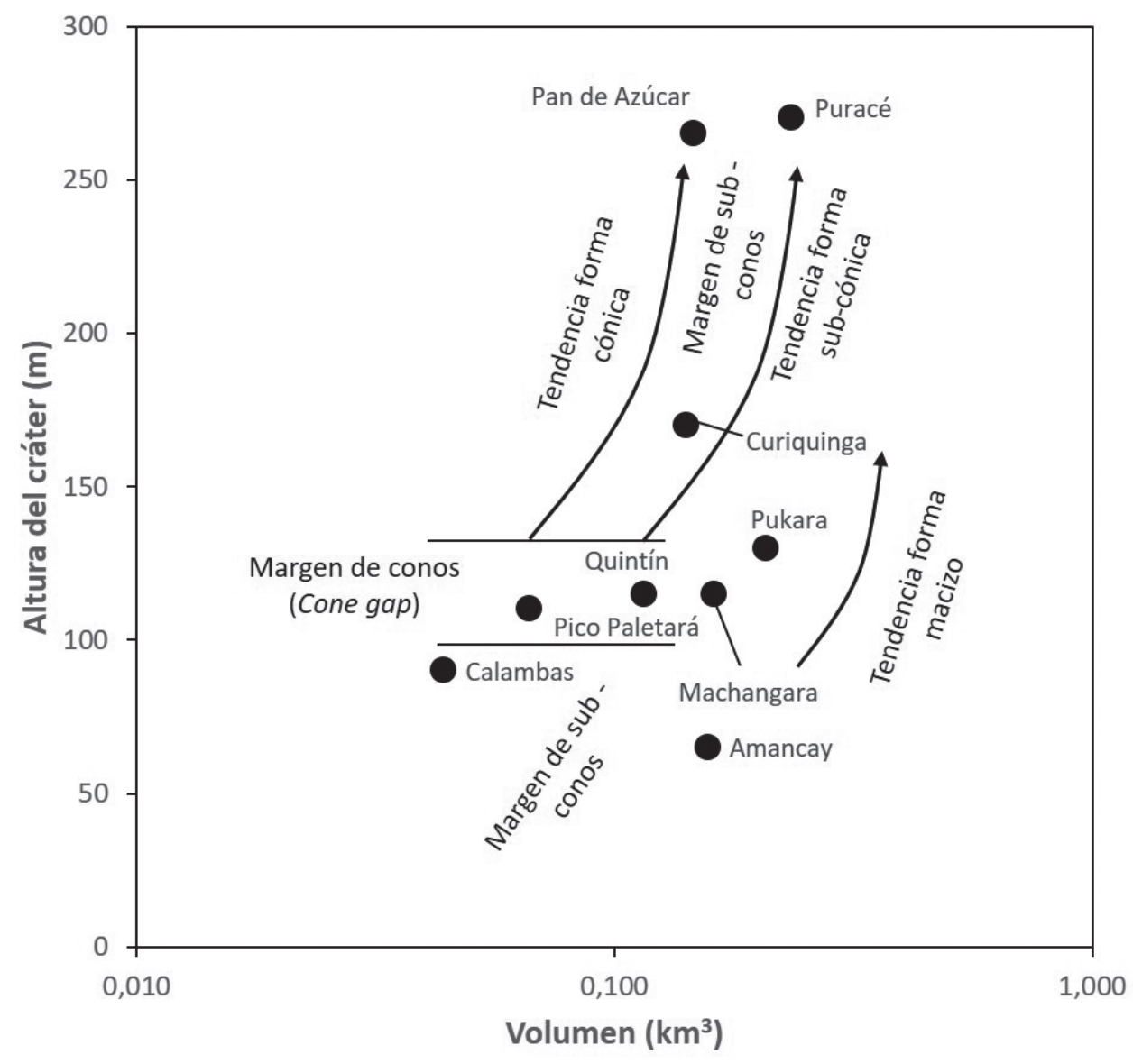

FIGURA 6. Diagrama de altura de cráter $v s$. volumen para clasificar los tipos de volcanes compuestos identificados en la CVLC. Adaptado de Grosse et al. (2009).

Los volcanes Pan de Azúcar y Puracé, con posiciones espaciales contrastantes en la CVLC, llaman la atención porque sus productos (TABLA 1) y sus características morfológicas (escasa disección por escorrentía laminar, casi nula afectación por acción de procesos glaciares) sugieren una construcción reciente en ambos casos (FIGURA 7), aunque el Puracé es el único volcán activo. 
A.

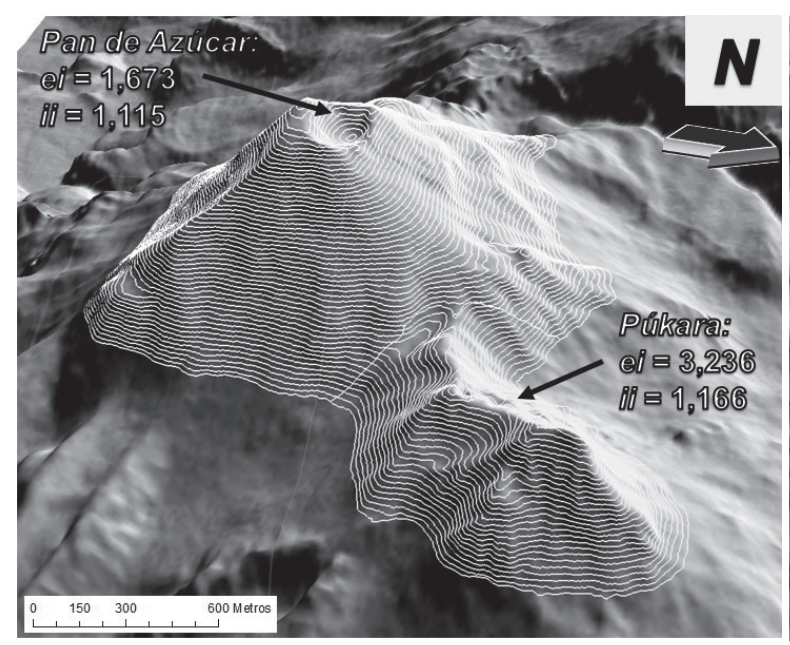

B.

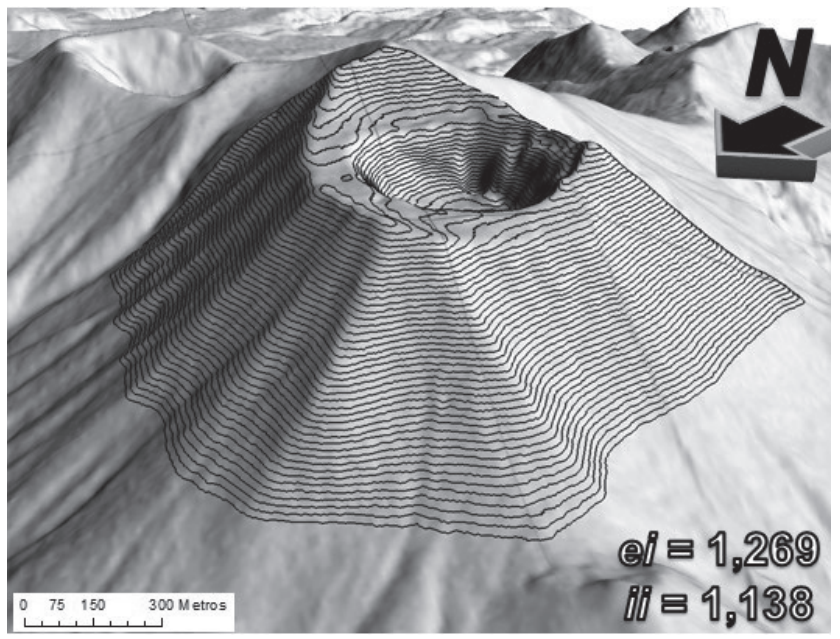

FIGURA 7. A. Vista del modelo de sombras en perspectiva oblicua de los volcanes compuestos Púkara y Pan de Azúcar con sus respectivos valores de $e_{i}$ e $i_{i}$. B. Vista del volcán compuesto Puracé. Los contornos topográficos mostrados en ambos casos son los utilizados en la parametrización morfométrica.

Otras estructuras ubicadas en la zona central de la CVLC (como Quintín y Machángara) muestran morfologías conservadas, a pesar de que no han presentado procesos de actividad reciente (FIGURA 8).
A.

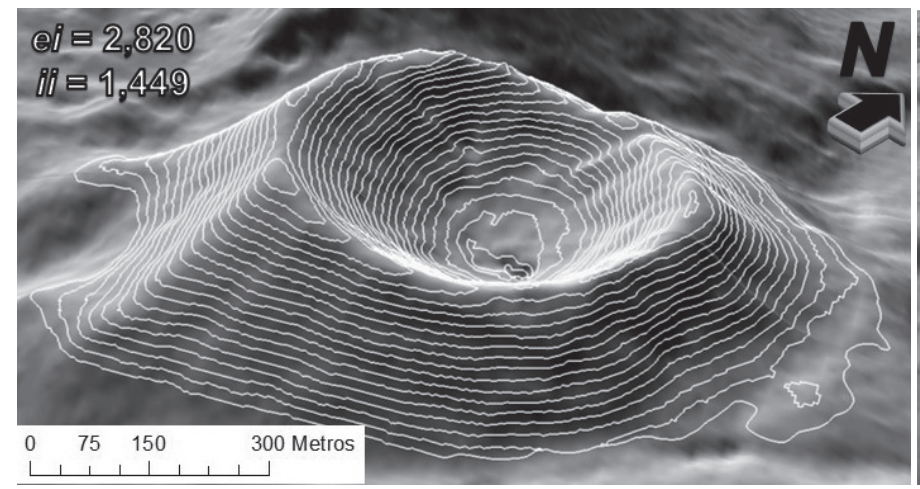

B.

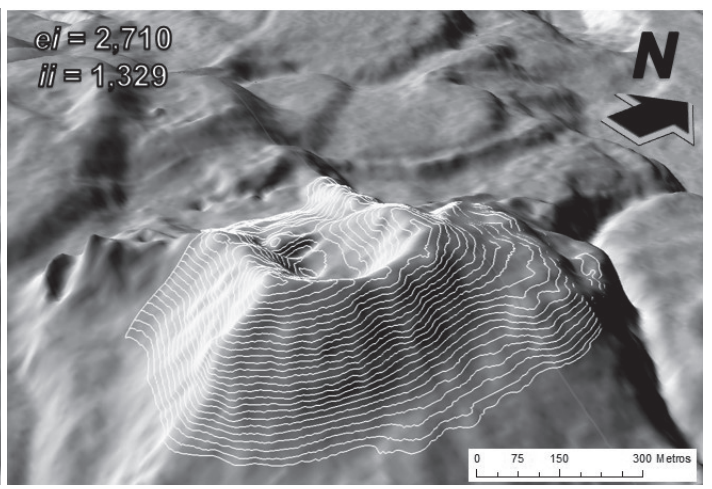

FIGURA 8. Vistas del modelo de sombras en perspectiva oblicua de los volcanes compuestos Quintín A y Machángara B. Convenciones como en la FIGURA 7.

Para los volcanes monogenéticos de la CVLC, que han sido tradicionalmente considerados como conos (TABLA 1), existe variabilidad en el valor de $H_{c o} /$ $W_{c o}$ que puede indicar diferencias en sus procesos de desarrollo o en sus tiempos de evolución y crecimiento. Se identifica de manera clara un patrón en los valores de la relación $H_{c o} / W_{c o}$, especialmente evidente al ordenar los datos de acuerdo a la coordenada de longitud del cono (FIGURA 5) el valor de $H_{c o} / W_{c o}$, con valores en el rango 0,017 (maar Hacienda Paletará, el más occidental) - 0,340 (Cono Piki, el más oriental) crece de manera regular de $\mathrm{W}$ a E. Este resultado, aunque intuitivo para volcanes relativamente aislados y de forma suavizada como El Canelo y Hacienda Paletará (FIGURA 1), si sorprende para volcanes como Piki, Shaka 1, 2 y 3, y Killa que están relativamente agrupados y presentarían influencias mutuas en sus morfologías, especialmente en el caso del grupo Shaka 1,2 y 3 y Killa. Estos conos aglomerados presentarían depósitos de piroclastos y emplazamientos de lavas superpuestos que enmascararían sus características morfológicas mutuas. Por otra parte, es posible que la proximidad espacial de algunos volcanes y la actividad cercana en el tiempo representen ciclos de actividad 
común como sería el caso de los conos Shaka 1,2 y 3 , volcanes monogenéticos que tienen productos similares y más recientes en comparación con sus similares de la CVLC (INGEOMINAS, 2003) (FIGURA 9).

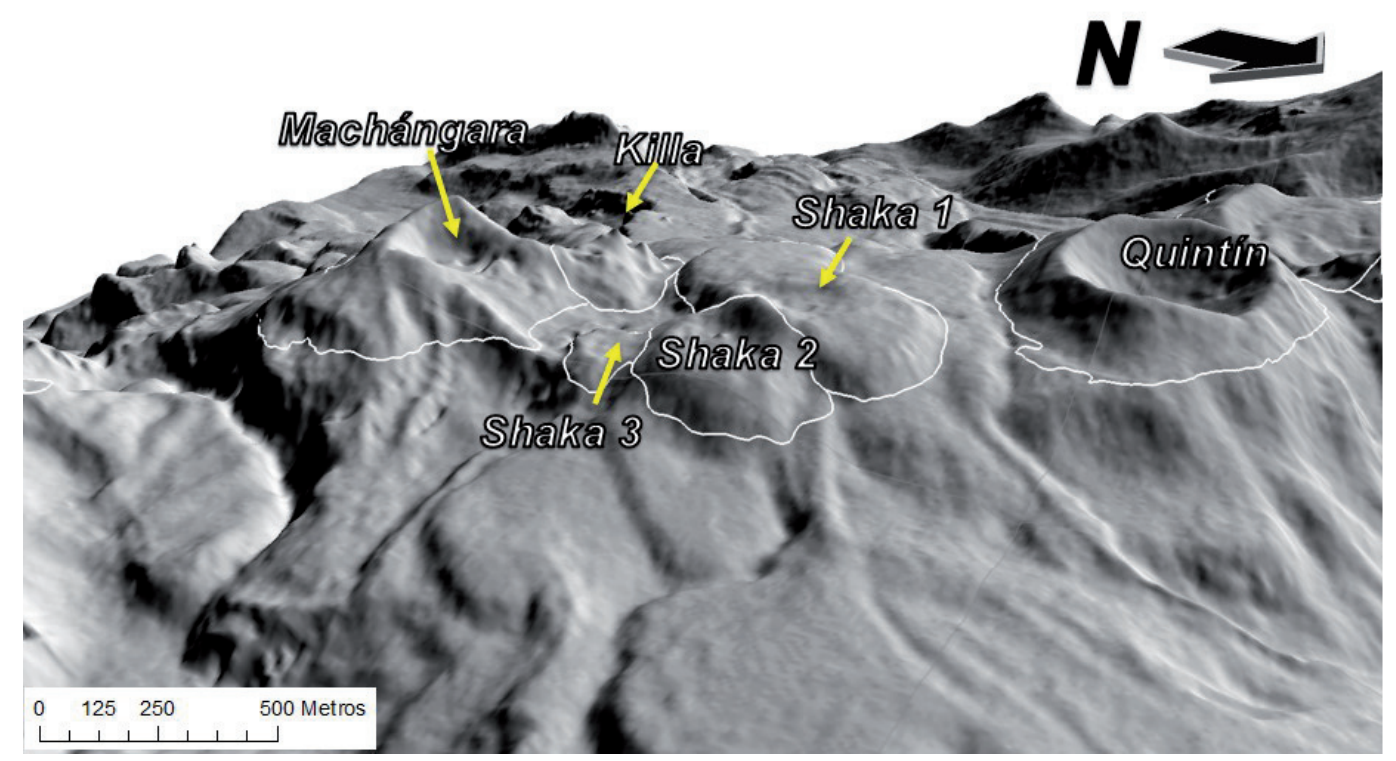

FIGURA 9. Vista del modelo de sombras en perspectiva con la agrupación de conos de escoria Shaka 1, 2 y 3, el volcán Killa y los volcanes compuestos Quintín y Machángara. Los contornos reflejan la delimitación de la base de cada estructura.

El índice $H_{c o} / W_{c o}$ define valores de estabilidad morfológica y los procesos geomorfológicos son inherentes a cada una de las estructuras (Settle, 1979), por lo que es difícil aproximar un mismo estilo y edad relativa de proceso eruptivo para los volcanes monogenéticos. De cualquier modo, es evidente que hay una progresión espacial suave de los valores de $H_{c o} / W_{c o}$ entre estos volcanes, lo cual demuestra la ventaja del método de análisis presentado aquí, en el sentido de revelar detalles y patrones que escaparían a una simple inspección visual o incluso a análisis espaciales computarizados.

Finalmente se sugiere que la variabilidad de morfologías de los centros volcánicos de la CVLC $\mathrm{y}$ vecindades representa historias muy diversas de formación y evolución de los volcanes compuestos y conos, hecho que es particularmente llamativo para centros volcánicos aglomerados y relacionados a una estructura fisural (i?) como el Lineamiento de Los Coconucos. Una estructura mayor denominada la Caldera de Paletará (Torres et al., 1995; Samacá, 2016), rodea la CVLC, y los aspectos presentados en este trabajo indican que la resurgencia magmática es definitivamente un conjunto de procesos complejo que merece futuras investigaciones tanto regionales como detalladas.

\section{CONCLUSIONES}

Se realizó el cálculo y análisis de parámetros morfométricos para 16 centros volcánicos de la CVLC y vecindades. Nueve centros volcánicos son morfológicamente clasificables como volcanes compuestos: Puracé, Curiquinga, Calambas, Amancay, Pico Paletará, Quintín, Machángara, Púkara y Pan de Azúcar. Cinco estructuras son clasificables como volcanes monogenéticos: Piki, Shaka 1, Shaka 2, Shaka 3 y Killa. Dos volcanes localizados al SW de la CVLC son clasificables como maares: El Canelo y Hacienda Paletará.

Los índices de elipticidad $\left(e_{i}\right)$ e irregularidad $\left(i_{i}\right)$ y la relación $H / W_{B}$ para volcanes compuestos varían así: 1,269 (Puracé) $\leq e_{i} \leq 3,236$ (Púkara); 1,115 (Pan de Azúcar) $\leq i_{i} \leq 1,530$ (Calambas); 0,044 (Calambas) $\leq H / W_{B} \leq 0,235$ (Pan de Azúcar). La relación $H_{c o}{ }^{\prime}$ $W_{c o}$ para volcanes monogenéticos y maares varía en el rango 0,017 (maar Hacienda Paletará) $\leq H_{c o} / W_{c o} \leq$ 0,340 (cono Piki).

Aunque los valores de $e_{i}$ e $i_{i}$ exhiben carácter oscilante en gráficos de acuerdo a las coordenadas de latitud y longitud, hay patrones incipientes de crecimiento o disminución en estos parámetros. Los volcanes 
compuestos de la CVLC se hacen más elongados $\mathrm{y}$ menos irregulares hacia el SE. De modo similar, la relación espacialmente variable $H / W_{B}$ presenta aumento general hacia el SE. El patrón de variación espacial del parámetro morfométrico $H_{c o} / W_{c o}$ para volcanes monogenéticos es muy marcado, especialmente al ordenar los valores de acuerdo a la coordenada de longitud. Este parámetro aumenta de manera gradual hacia el NE.

\section{AGRADECIMIENTOS}

Los autores agradecen a la Subdirección de Geografía y Cartografía del Instituto Geográfico Agustín Codazzi (IGAC) por facilitar las imágenes de radar necesarias para el desarrollo de este trabajo. Las revisiones de la Dra. Elmilce Bustos (INENCO-Argentina) y el Dr. Hugo Murcia (IIES-Universidad de Caldas, Colombia) ayudaron a mejorar la calidad de este artículo.

\section{REFERENCIAS}

De Silva, S., and Lindsay, J.M. (2015). Primary Volcanic Landforms. In: H. Sigurdsson, B. Houghton, S. McNutt, H. Rymer, and J. Stix (Ed.). Encyclopedia of Volcanoes (pp. 273-297). 2nd Edition. Academic Press.

Favalli, M., Karátson, D., Mazzarini, F., Pareschi, M.T., and Boschi, E. (2009). Morphometry of scoria cones located on a volcano flank: A case study from Mt. Etna (Italy) based on highresolution LiDAR data. Journal of Volcanology and Geothermal Research, 186(3-4), 320-330. doi: 10.1016/j.jvolgeores.2009.07.011.

Flórez, A. (1983). Cadena volcánica de los Coconucos, Cordillera Central, Colombia. Revista Colombia Geográfica, X, 33-53.

Flórez, A. (2003). Colombia, evolución de sus relieves y modelados. Bogotá: Universidad Nacional de Colombia, UNIBIBLOS.

Karátson, D., Favalli, M., Tarquini, S., Fornaciai, A., and Wörner, G. (2010). The regular shape of stratovolcanoes: A DEM-based morphometrical approach. Journal of Volcanology and Geothermal Research, 193(3-4), 171-181. doi: 10.1016/j. jvolgeores.2010.03.012.
Grosse, P., van Wyk de Vries, B., Petrinovic, I.A., Euillades, P.A. and Alvarado, G.E. (2009). Morphometry and evolution of arc volcanoes. Geology, 37(7), 651-654. doi: 10.1130/ G25734A.1.

Grosse, P., van Wyk de Vries, B., Euillades, P.A., Kervyn, M., and Petrinovic, I.A. (2012). Systematic morphometric characterization of volcanic edifices using digital elevation models. Geomorphology, 136(1), 114-131. doi: 10.1016/j. geomorph.2011.06.001.

INGEOMINAS. (2003). Geología de la Plancha 365 - Coconuco. Mapa Geológico escala 1:100.000 y Memoria Explicativa.

Monsalve, M.L. (2000). Catálogo de las unidades litoestratigráficas de Colombia, Formación Coconucos. INGEOMINAS, $31 \mathrm{p}$.

Monsalve, M.L., Galarza, J.J., Zuluaga, I., y Laverde, C.A. (2014). Mapa de amenaza volcánica del volcán Puracé actual (Cauca-Colombia) segunda versión. Servicio Geológico Colombiano, 49 p.

Porter, S. (1972). Distribution, morphology and size of cinder cones on Mauna Kea volcano, Hawaii. Geological Society of America Bulletin, 83(12), 3607-3612. doi: 10.1130/0016-7606(1972)83[3607:DMASFO]2. $0 . \mathrm{CO} ; 2$.

Robertson, K., Flórez, A., y Ceballos, J.L. (2002). Geomorfología volcánica, actividad reciente y clasificación en Colombia. Cuadernos de Geografia: Revista Colombiana de Geografía, $11,37-76$.

Settle, M. (1979). The structure and emplacement of cinder cones fields. American Journal of Science, 279(10), 1089-1107. doi: 10.2475/ ajs.279.10.1089.

Samacá, W. (2016). Análisis morfométrico y geomorfológico de la Caldera de Paletará (Cauca), Colombia. Tesis de Maestría, Universidad Nacional de Colombia, Bogotá. 
Torres, M.P., Monsalve, M.L., Pulgarín, B., y Cepeda, H. (1995). Caldera de Paletará: aproximación a la fuente de las ignimbritas del Cauca y Huila (Colombia). INGEOMINAS, informe interno, 20 p.

\begin{tabular}{c}
\hline \hline Wveimar Samacá \\
ORCID: 0000-0003-4032-8548 \\
John J. Sánchez \\
ORCID: 0000-0002-9024-7554 \\
\hline \hline
\end{tabular}

Wood, C.A. (1980). Morphometric analysis of cinder cone degradation. Journal of Volcanology and Geothermal Research, 8(2-4), 137-160. doi: 10.1016/0377-0273(80)90101-8.

Trabajo recibido: noviembre 16 de 2017

Trabajo aceptado: mayo 17 de 2018 